\title{
Discrete-time Holling type II models with Allee and refuge effects
}

\author{
Unal Ufuktepe and Burcin Kulahcioglu \\ Communicated by M. Zuhair Nashed
}

\begin{abstract}
The discrete-time Holling type II prey-predator models with the refuge and Allee effects are formulated and studied. The existence of fixed points and their stabilities are investigated for both hyperbolic and non-hyperbolic cases. Numerical simulations are conducted to demonstrate the theoretical results.
\end{abstract}

Keywords. Holling type II, prey-predator, refuge effect.

2010 Mathematics Subject Classification. 92B05, 37C75, 37C25.

\section{Introduction}

Predator-prey models are used to describe naturally occurring biological or ecological relationships between two interacting species. These models have been studied extensively by researchers from the fields of mathematical biology and mathematical ecology. One of the important predator-prey models is Holling type II model. The real life application of this model is the Lynx and its prey the snowshoe Hare study documented by the Hudson Bay company for the time interval 1845-1935 [2]. There is a little attention to the discrete case because of the complexities of this model. In this study, we focus on analyzing how the Holling type II response [6] affects the dynamic complexities of prey predator interactions with Allee and refuge effects.

We organize this paper as follows: In Section 2, we incorporate a refuge effect into the discrete prey predator model with Holling type II, then we show the existence and stability of fixed points. We use The Center Manifold Theorem for the non-hyperbolic fixed point. We present some numerical simulations of the analytic results to compare them with the results of [1]. In Section 3, we incorporate an Allee effect into the discrete prey predator model with Holling type II, then we show the existence and stability of fixed points. Some numerical simulations of

This study was supported by The Scientific and Technological Research Council of Turkey (TÜBİTAK). 
the analytic results are presented to compare them with the results of [1]. Some values of the parameters for which the model undergoes the Neimark-Sacker bifurcation in the positive region, are derived. Also, the global behavior of the model is discussed. Finally, in Section 4 we draw conclusions.

The following prey-predator model with the Holling type II functional response [7] has been studied by many authors $[1,6,9]$.

$$
\begin{aligned}
x^{\prime} & =a x(1-x)-\alpha \frac{m x y}{1+\varepsilon x}, \\
y^{\prime} & =\left(\frac{m x}{1+\varepsilon x}-\beta\right) y,
\end{aligned}
$$

where all the parameters are positive, and

- $\alpha$ is conversion,

- $\beta$ is predator's death rates,

- $a$ is prey intrinsic growth parameter,

- $m$ is half saturation parameter,

- $\varepsilon$ is limitation of the growth velocity of the predator population with increase in the number of prey.

We refer to the works in $[4,9,11]$. Equilibrium points of this model are stable or limit cycles.

The discrete prey-predator model with Holling type II studied by Agiza et al. [1] is as follows:

$$
\begin{aligned}
x_{n+1} & =a x_{n}\left(1-x_{n}\right)-\frac{b x_{n} y_{n}}{1+\varepsilon x_{n}}, \\
y_{n+1} & =\frac{d x_{n} y_{n}}{1+\varepsilon x_{n}},
\end{aligned}
$$

where all parameters are non-negative. This map is non-invertible map.

We first study this model with a fixed number of prey in refuge, then with an Allee effect. The study of the dynamical properties of the resulting models allows us to have information about the future behavior of prey predator populations.

\section{Holling type II model with a refuge effect}

The study of the consequences of hiding behavior of prey on the dynamics of predator prey interactions can be recognized as a major issue in applied mathematics and theoretical ecology. A simple prey response to predators involves the 
use of refuges: places or situations where predation risk is somehow reduced. The traditional ways by which the effects of prey refuges have been incorporated in predator-prey interactions is to consider two types of refuges in the literature: those that protect a constant fraction of prey and those that protect a constant number of prey $[5,8,10,12,13]$. We study the following model obtained by adding a refuge effect $R$ to prey in the model given by [1]:

$$
\begin{aligned}
& x_{n+1}=a x_{n}\left(1-x_{n}\right)-\frac{b\left(x_{n}-R\right) y_{n}}{1+e\left(x_{n}-R\right)}, \\
& y_{n+1}=\frac{d\left(x_{n}-R\right) y_{n}}{1+e\left(x_{n}-R\right)},
\end{aligned}
$$

where $a, b, d, e, R$ are positive.

\subsection{Fixed points of the map defined by (3) and their stability}

In this section, we investigate the fixed points of the map defined by (3) and their stability conditions. The solution of the following system of equations gives us three non-negative fixed points:

$$
\begin{aligned}
& x=a x(1-x)-b \frac{(x-R) y}{1+e(x-R)}, \\
& y=\frac{d(x-R) y}{1+e(x-R)} .
\end{aligned}
$$

The fixed points are :

a. for any values of parameters, there exists extinction fixed point $E_{0}=(0,0)$.

b. there exists one exclusion fixed point $E_{1}=\left(\frac{a-1}{a}, 0\right)$ for $a>1$.

c. there exists one positive fixed point $E_{2}=\left(\frac{\Phi}{Q},-\frac{d \Phi(Q+a \Phi-a Q)}{b Q^{2}}\right)$ for

- $Q<\Phi<0$ and $a>\frac{Q}{Q-\Phi}$, or

- $0<\Phi<Q$ and $a>\frac{Q}{Q-\Phi}$,

where $Q=d-e$ and $\Phi=1+R Q$.

The Jacobian matrix of the map (3) is as follows:

$$
J(x, y)=\left(\begin{array}{cc}
-2 x a+a-\frac{b y}{(e(x-R)+1)^{2}} & \frac{b(x-R)}{e(R-x)-1} \\
\frac{d y}{(e(x-R)+1)^{2}} & \frac{d(x-R)}{e(x-R)+1}
\end{array}\right) .
$$


For the fixed point $E_{0}$,

$$
J\left(E_{0}\right)=\left(\begin{array}{cc}
a & \frac{b R}{1-e R} \\
0 & \frac{d R}{e R-1}
\end{array}\right)
$$

The eigenvalues are $\lambda_{1}=a$ and $\lambda_{2}=\frac{d R}{e R-1}$. The fixed point $E_{0}$ is hyperbolic if neither of these eigenvalues is on the unit circle, that is, $\left|\lambda_{1,2}\right| \neq 1$. In addition, $E_{0}$ is a stable fixed point (sink) if $a<1$ (the map has only one non-negative fixed point), $d<\frac{e R-1}{R}$, and $R>\frac{1}{e}$. Also, if $a<1, d<\frac{1-e R}{R}$, and $e<\frac{1}{R}$, then $E_{0}$ is stable (oscillatory). If $a=1$ or $d=\frac{e R-1}{R}$ or $d=\frac{1-e R}{R}$, then one of the eigenvalues of $J\left(E_{0}\right)$ lies on the unit circle, that is, $|\lambda|=1$. As a result, for these values of parameters, the fixed point is non-hyperbolic.

We consider only the case $a=1$. We need to write the system in the following form to use the Center Manifold Theorem:

$$
\begin{aligned}
& x_{n+1}=A x_{n}+f\left(x_{n}, y_{n}\right), \\
& y_{n+1}=B y_{n}+g\left(x_{n}, y_{n}\right) .
\end{aligned}
$$

Furthermore $f(0,0)=0, g(0,0)=0, D f(0,0)=0, D g(0,0)=0$. Let $M_{c}=$ $\{(x, y) \in R x R: y=h(x), h(0)=D h(0)=0\}$. If we substitute the parameters $a=1, b=0.2, d=3.5, e=0.9$, and $R=1 / 9$, then we obtain the following Jacobian matrix at $E_{0}$ :

$$
J(0,0)=\left(\begin{array}{rr}
1 & 0.0246914 \\
0 & -0.432099
\end{array}\right) .
$$

Hence, the fixed point $E_{0}=(0,0)$ is non-hyperbolic. Now, the system (3) can be written as

$$
\begin{aligned}
& x_{n+1}=x_{n}+0.0246914 y_{n}+f\left(x_{n}, y_{n}\right), \\
& y_{n+1}=-0.432099 y_{n}+g\left(x_{n}, y_{n}\right),
\end{aligned}
$$

where

$$
f(x, y)=-x^{2}-0.0246914 y-\frac{0.2 y\left(x-\frac{1}{9}\right)}{1+0.9\left(x-\frac{1}{9}\right)},
$$

and

$$
g(x, y)=0.432099 y+\frac{3.5 y\left(x-\frac{1}{9}\right)}{1+0.9\left(x-\frac{1}{9}\right)} .
$$

Let

$$
h(x)=c_{1} x^{2}+c_{2} x^{3}+O\left(x^{4}\right),
$$


for $c_{1}, c_{2} \in \mathbb{R}$. In order to compute the constants $c_{1}$ and $c_{2}$, the following functional equation should be solved:

$$
\begin{aligned}
h\left(x_{n}\right. & \left.+0.0246914 h\left(x_{n}\right)+f\left(x_{n}, h\left(x_{n}\right)\right)\right) \\
& =-0.432099 h\left(x_{n}\right)+g\left(x_{n}, h\left(x_{n}\right)\right) .
\end{aligned}
$$

By solving equation (10), we find $c_{1}=c_{2}=0$. Now, on the center manifold $y=h(x)$, we obtain the following one dimensional map:

$$
x_{n+1}=x_{n}-x_{n}^{2}+O\left(x_{n}^{4}\right) .
$$

Let $f(x)=x-x^{2}+O\left(x^{4}\right)$. Since $f^{\prime}(0)=1$ and $f^{\prime \prime}(0)=-2<0$, by the Schwarzian derivative and Center Manifold theorems [3], the origin is semistable from right under the given set of parameters (Similarly, we get the same results for $d=\frac{e R-1}{R}$ and $d=\frac{1-e R}{R}$ ).

For the fixed point $E_{1}$,

$$
J\left(E_{1}\right)=\left(\begin{array}{cc}
2-a & \frac{b(-R a+a-1)}{e+a(e(R-1)-1)} \\
0 & \frac{d(a(R-1)+1)}{e+a(e(R-1)-1)}
\end{array}\right) .
$$

The eigenvalues are $\lambda_{1}=2-a$ and $\lambda_{2}=\frac{d(a(R-1)+1)}{e+a(e(R-1)-1)}$. Then $E_{1}$ is a stable fixed point (sink) if $1<a<3$ and $\left|\lambda_{2}\right|<1$. Otherwise, it is unstable. If $a=3$ or $d=\frac{1+a(R-2)}{1+a(R-1)}$, then similarly, the center manifold theorem must be used as above. For the fixed point $E_{2}$,

$$
J\left(E_{2}\right)=\left(\begin{array}{cc}
a-\frac{2 a p}{Q}+\frac{d p(a(p-Q)+Q)}{(Q+e(p-Q R))^{2}} & -\frac{b}{d} \\
-\frac{d^{2} p(a(p-Q)+Q)}{b(Q+e(p-Q R))^{2}} & 1
\end{array}\right) .
$$

The positive fixed point is stable if the eigenvalues of the Jacobian matrix are $\left|\lambda_{1,2}\right|<1$. This case will be investigated in the numerical simulations section.

We write some codes with Mathematica 10 for the calculation of non-negative fixed points under some conditions of parameters, for simulations of phase diagrams, and time series in Numerical Simulations sections.

\subsection{Numerical simulations for the model (3)}

We first study the model when we vary only the parameter $R$ (refuge effect) and fix the others with respect to the above analytical results. Second, we study the model (3) for varying values of the parameter e. $E_{1}$ and $E_{2}$ are not in the positive region if $0<a<1$. There is no biological meaning when the trajectory of the 
map is oscillating on the positive and negative regions for $0<a<1$. Thus, we will give the numerical simulation only when $a>1$ and $d>e$.

Without loss of the generality, we fix the parameters $a=4, b=0.2, d=$ $3.5, e=0.1$ and assume $R$ varies. Under this set of parameter values, $E_{2}$ is a spiral sink if $0.09<R<0.24$, and it is an oscillatory saddle if $0.24<R<0.46$. For example, if $R=1 / 9$, the fixed points are $E_{0}=(0,0), E_{1}=(0.75,0)$ and $E_{2}=(0.405229,9.77979)$ with the eigenvalues $\lambda_{E_{0}}=\{4 .,-0.393258\}, \lambda_{E_{1}}=$ $\{-2 ., 2.10183\}$, and $\lambda_{E_{2}}=\{-0.0438074 \pm 0.869627 i\}$, respectively. In this case $E_{0}$ is an oscillatory saddle, $E_{1}$ is an oscillatory source, and $E_{2}$ is a stable focus (spiral sink).

Next, we fix the parameters $a=4, b=0.2, d=3.5, R=1 / 9$ and assume $e$ varies. The positive fixed point $E_{2}$ is a spiral sink if $0<e<0.92$, it is an oscillatory sink if $0.92<e<1.45$, otherwise it is unstable. For example, the fixed points are $E_{0}=(0,0), E_{1}=(0.75,0)$ and $E_{2}=(0.495726,8.82351)$ when $e=0.9$. The eigenvalues are $\lambda_{E_{0}}=\{4 .,-0.432099\}, \lambda_{E_{1}}=\{-2 ., 1.41975\}$, and $\lambda_{E_{2}}=\{0.0301804 \pm 0.18242 i\}$, respectively. In this case $E_{0}$ is an oscillatory saddle, $E_{1}$ is an oscillatory source, and $E_{2}$ is a spiral sink. We give some phase portraits for different values of $e$ (Figure 1, and Figure 2).

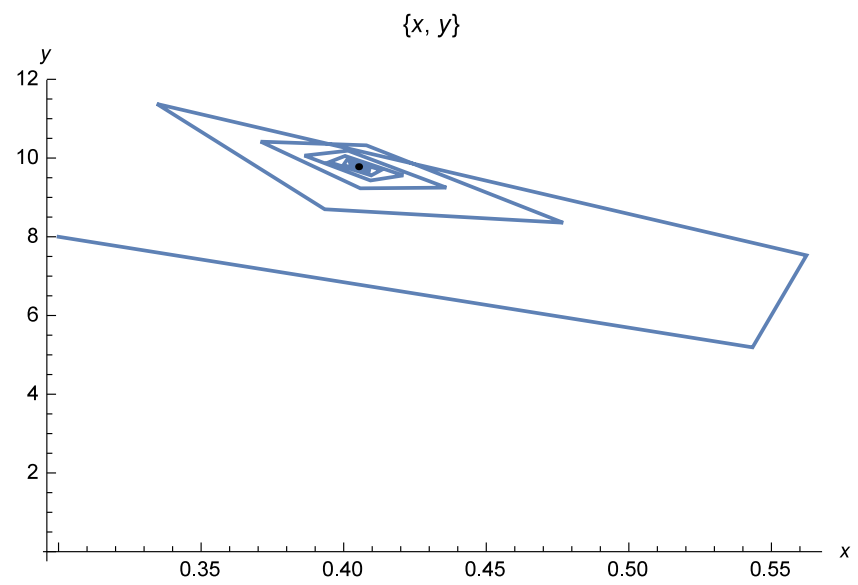

Figure 1. Phase portrait for the model (3) for $e=0.1$

\section{Holling type II model with an Allee effect}

The Allee effect occurs when positive density-dependence dominates at low densities. When the Allee effect is sufficiently strong, there is a critical threshold below 


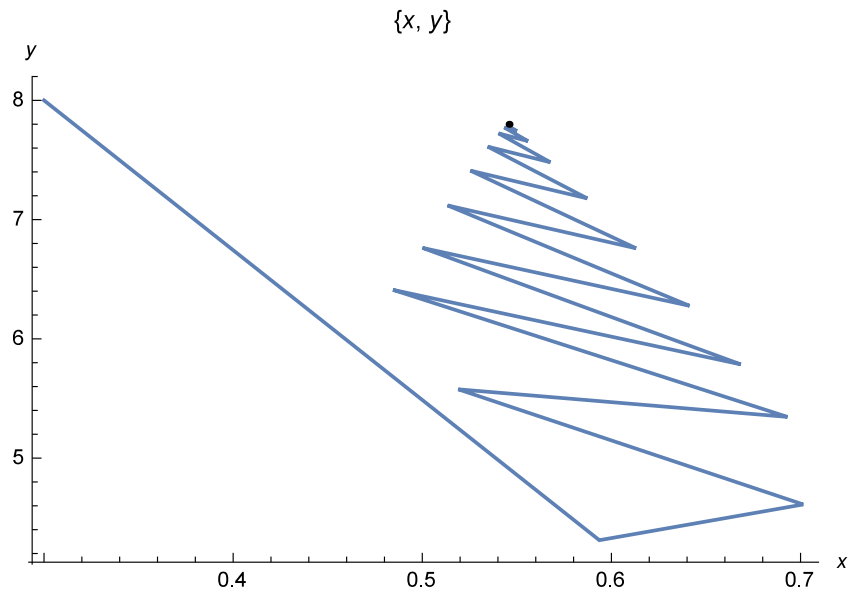

Figure 2. Phase portrait for the model (3) for $e=1.2$

which populations experience rapid extinction. Consequently, the importance of the Allee effect has been widely recognized in conservation biology.

For many sexually reproducing organisms, finding mates becomes more difficult at low densities. Species with low dispersal rates are less likely to encounter mates at small population sizes.

Let $I(x)=\frac{x}{x+K}$ be the probability of finding a mate where $1 / K>0$ is an individual's searching efficiency. We study the following model which is given by [1] by adding the mate limitation Allee effect to the prey:

$$
\begin{aligned}
x_{n+1} & =\left(a x_{n}\left(1-x_{n}\right)-\frac{b x_{n} y_{n}}{1+e x_{n}}\right) \frac{x_{n}}{K+x_{n}}, \\
y_{n+1} & =\frac{d x_{n} y_{n}}{1+e x_{n}}
\end{aligned}
$$

where $a, b, d, e, K$ are positive.

\subsection{Fixed points of the map defined by (12) and their stability}

In this section, we investigate the fixed points of the map defined by (12) and their stability conditions. The solution of the following system of equations gives us 
four non-negative fixed points:

$$
\begin{aligned}
& x=\left(a x(1-x)-b \frac{x y}{1+e x}\right) \frac{x}{K+x}, \\
& y=\frac{d x y}{1+e x} .
\end{aligned}
$$

The fixed points are

a. for any values of parameters, there exists extinction fixed point $A_{0}=(0,0)$,

b. there exist two exclusion fixed points $A_{1,2}=\left(\frac{a-1 \pm M}{2 a}, 0\right)$ if $a>1$ and $K<$ $\frac{(a-1)^{2}}{4 a}$,

c. there exists one positive fixed point $A_{3}=\left(\frac{1}{Q}, \frac{d(a(Q-1)-Q(1+Q K))}{b Q^{2}}\right)$ for $Q>$ 1 and $a>\frac{Q(1+K Q)}{Q-1}$, where $M=\sqrt{(a-1)^{2}-4 a K}$ and $Q=d-e$.

The Jacobian matrix of the map (12) is given by

$$
J(x, y)=\left(\begin{array}{cc}
-\frac{x\left(a(x(2 x-1)+K(3 x-2))(e x+1)^{2}+b(x+K(e x+2)) y\right)}{(K+x)^{2}(e x+1)^{2}} & -\frac{b x^{2}}{(K+x)(e x+1)} \\
\frac{d y}{(e x+1)^{2}} & \frac{d x}{e x+1}
\end{array}\right) .
$$

For the fixed point $A_{0}$,

$$
J\left(A_{0}\right)=\left(\begin{array}{ll}
0 & 0 \\
0 & 0
\end{array}\right)
$$

$A_{0}$ is stable .

For the fixed point $A_{1}=\left(\frac{a-1-M}{2 a}, 0\right)$,

$$
J\left(A_{1}\right)=\left(\begin{array}{cc}
\frac{M+a(-a+6 K+M+4)-1}{2 a(K+1)} & -\frac{b(-a+M+1)^{2}}{(-a(2 K+1)+M+1)(M e+e-a(e+2))} \\
0 & \frac{d(-a+M+1)}{M e+e-a(e+2)}
\end{array}\right) .
$$

If $\left|\frac{M+a(-a+6 K+M+4)-1}{2 a(K+1)}\right|<1$ and $\left|\frac{d(-a+M+1)}{M e+e-a(e+2)}\right|<1$, thus $A_{1}$ is a stable fixed point.

For the fixed point $A_{2}=\left(\frac{a-1+M}{2 a}, 0\right)$,

$$
J\left(A_{2}\right)=\left(\begin{array}{cc}
-\frac{M+a(a-6 K+M-4)+1}{2 a(K+1)} & -\frac{b(a+M-1)^{2}}{(2 K a+a+M-1)(a(e+2)+e(M-1))} \\
0 & \frac{d(a+M-1)}{a(e+2)+e(M-1)}
\end{array}\right) .
$$

If $\left|-\frac{M+a(a-6 K+M-4)+1}{2 a(K+1)}\right|<1$ and $\left|\frac{d(a+M-1)}{a(e+2)+e(M-1)}\right|<1$, thus $A_{2}$ is a stable fixed point.

For the fixed point $A_{3}$, 


$$
J\left(A_{3}\right)=\left(\begin{array}{cc}
\frac{(2 d K-e K+1) Q^{2}+a(d(e-1)-e(e+1))}{d Q(K Q+1)} & -\frac{b}{Q K d+d} \\
\frac{-K Q^{2}-Q+a(Q-1)}{b} & 1
\end{array}\right) .
$$

The positive fixed point is stable if the eigenvalues of the Jacobian matrix are $\left|\lambda_{1,2}\right|<1$. This case will be investigated in the numerical simulations section.

\subsection{Global behavior of model (12)}

The extinction fixed point $A_{0}$ of model (12) is stable for any values of parameters. By comparison we have

$$
x_{n+1}=\left(a x_{n}\left(1-x_{n}\right)-\frac{b x_{n} y_{n}}{1+e x_{n}}\right) \frac{x_{n}}{K+x_{n}}<a x_{n}\left(1-x_{n}\right)-\frac{b x_{n} y_{n}}{1+e x_{n}},
$$

and

$$
a x_{n}\left(1-x_{n}\right)-\frac{b x_{n} y_{n}}{1+e x_{n}}<a x_{n}\left(1-x_{n}\right)<a x_{n} .
$$

Hence, if $a<1$,

$$
\lim _{n \rightarrow \infty} x_{n}=0 .
$$

Since $y_{n+1}=\frac{d x_{n} y_{n}}{1+e x_{n}}$,

$$
\lim _{n \rightarrow \infty} y_{n}=0
$$

which means $A_{0}$ is globally attracting and globally asymptotically stable if $a<1$. Consequently, if the growth parameter is small $(a<1)$, both populations become extinct even with large initial values. On the other hand, the other fixed points $A_{1}$, $A_{2}$ and $A_{3}$ cannot be globally stable because of the local stability of $A_{0}$.

\subsection{Numerical simulations for the model (12)}

We first study the model (12) when we change only the parameter $e$ and fix the others with respect to the above analytical results. We compare our results with the results of [1]. We fix the parameters $a=4.1, b=3, d=3.5, K=0.2$, and assume that $e$ varies as in [1]. When the control parameter $e$ varies in $(0,0.85)$, $(0.85,1.53)$ and $[1.53,2.03]$ the stability/unstability of positive fixed point changes through various types of bifurcations, but $A_{0}$ is always stable, and $A_{1,2}$ are always saddle in the positive region. $A_{3}$ is unstable (spiral source) if $0<e<0.85$. It is stable (spiral sink) if $0.85<e<1.53$, and it is stable (sink) if $1.53 \leq e \leq 2.03$. 
If $e>2.03$, it is not a positive fixed point anymore. We consider the following cases :

Let $e=1.9$ then $A_{0}=(0,0), A_{1}=(0.0712257,0), A_{2}=(0.684872,0)$ and $A_{3}=(0.625,0.158594)$ are the fixed points. Eigenvalues are $\lambda_{A_{1}}=\{1.66071,0.219575\}, \lambda_{A_{2}}=\{1.04163,-0.947291\}$ and $\lambda_{A_{3}}=\{0.951751,-0.561167\}$, respectively. Since $\left|\lambda_{A_{3}}\right|<1$, the positive fixed point is asymptotically stable (sink). $A_{1}$, and $A_{2}$ are saddles (Figure 3 ).

Let $e=0.86$, then the positive fixed point is $A_{3}=(0.378788,0.450302)$ (the others as same as in case $e=1.90$ ). Eigenvalues are $\lambda_{A_{3}}=\{0.746513 \pm$ $0.662383 i\}$. Since $\left|\lambda_{A_{3}}\right|=0.998015<1$, then the positive fixed point is stable (spiral sink) (Figure 4).

Now, let $e=0.85$, then the positive fixed point is $A_{3}=(0.377358,0.450303)$ (the others as same as in case $e=1.90$ ). We observe that the behavior of the model becomes very complicated including the Neimark-Sacker bifurcation. The fixed point $A_{3}$ loses its stability through a Neimark-Sacker bifurcation and an invariant closed curve is created around the fixed point when $e=0.85$ (Figure 5).

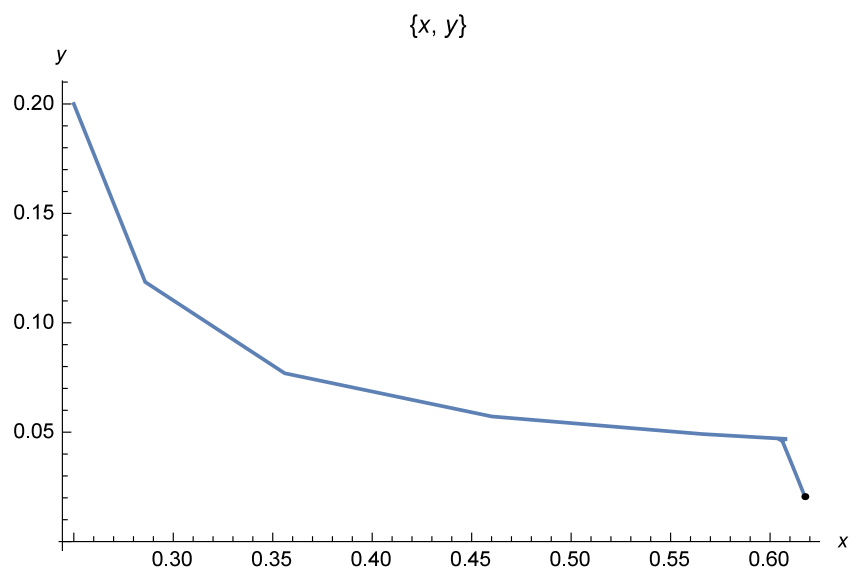

Figure 3. Phase portrait for the model (12) for $e=1.9$

When we compare these results with results of [1], we can see that Allee effect has a strong effect on the stability of system and changes the parameter intervals for stability. When $e$ is decreased, the behavior of the system changes to a chaotic one. We give the time series diagram of $x$ in Figure 6. The time series of $y$ is also similar but only the domain is different. 


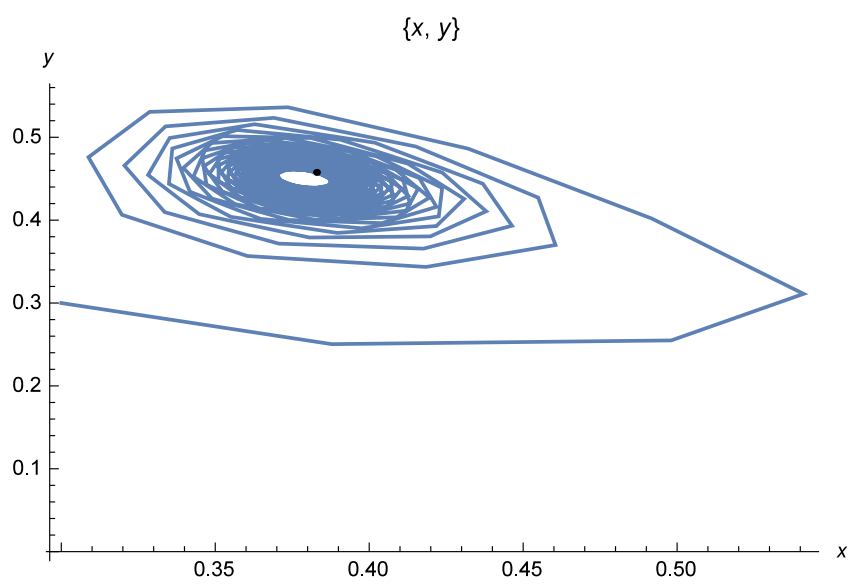

Figure 4. Phase portrait for the model (12) for $e=0.86$

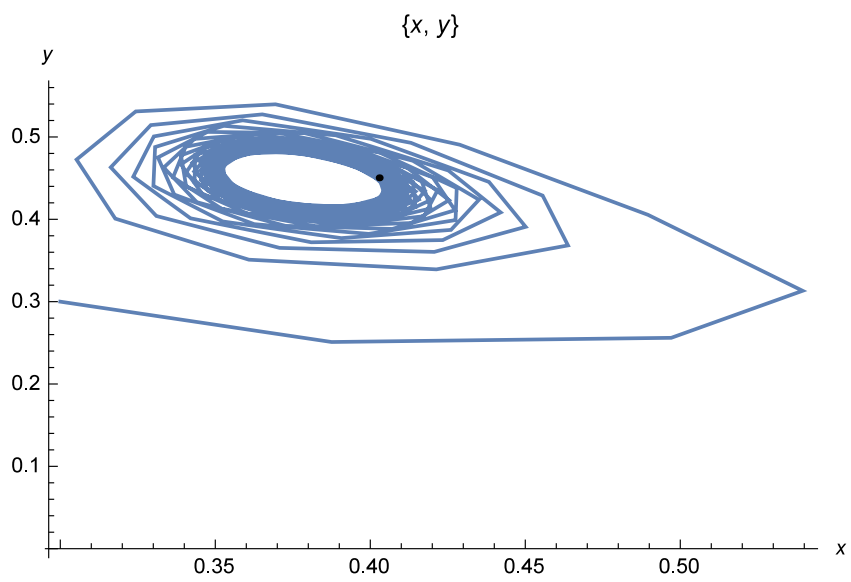

Figure 5. Phase portrait for the model (12) when a Neimark-Sacker bifurcation exists for $e=0.85$

\section{Conclusion}

We study these models since not only the discrete Holling type model has richer features and more complicated dynamics than in the continuous case, but also it is more realistic with an Allee effect and refuge effect than the model of [1]. The stability of the fixed points and bifurcations are analyzed. We show that the NeimarkSacker bifurcation parameter value $e$ is changed with Refuge and Allee effects. We find the intervals of $e$ where the positive fixed point is stable or unstable. We 


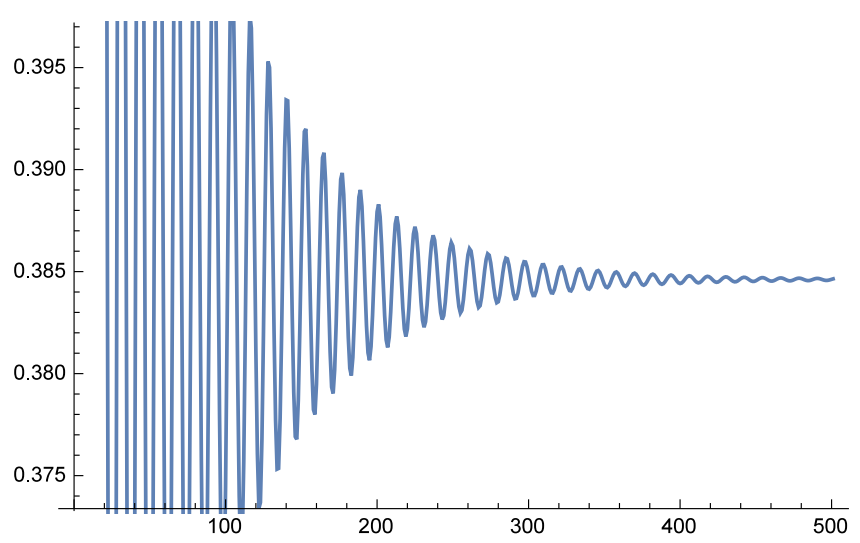

Figure 6. Time series of $x$ for the model (12) for $e=0.9$

use the Center Manifold Theorem to investigate whether the non-hyperbolic fixed point is stable or not. We see that both the mating limitation Allee effect and Refuge effect have strong impacts on the stability of the system.

\section{Bibliography}

[1] H. N. Agiza, E. M. ELabbasy, H. EL-Metwally and A. A. Elsadany, Chaotic dynamics of a discrete prey-predator model with Holling type II, Nonlinear Analysis: Real World Applications 10 (2009), 116-129.

[2] L. Edelstein-Keshet, Mathematical Models in Biology, Random House, New York, 1988.

[3] S. Elaydi, Discrete Chaos: With Applications in Science and Engineering, Second Edition, Chapman \& Hall/CRC, 2008.

[4] J. Hainzl, Stability and Hopf bifurcation in a predator-prey system with several parameters, SIAM J. Appl. Math. 48 (1988), 170-180.

[5] M. E. Hochberg and R. D. Holt, Refuge evolution and the population dynamics of coupled host-parasitoid associations, Evol. Ecol. 9 (1995), 633-661.

[6] C. S. Holling, The functional response of predator to prey density and its role in mimicry and population regulation, Mem. Ent. Soc. Canada 97(S45) (1965), 5-60.

[7] S. B. Hsu and T. W. Hwang, Global stability for a class predator-prey system, SIAM J. Appl. Math. 55 (1995), 763-783.

[8] T. K. Kar, Stability analysis of a prey-predator model incorporating a prey refuge, Commun. Nonlinear Sci. Numer. Simul. 10 (2005), 681-691. 
[9] N. Kasarinoff and P. van der Deiesch, A model of predator-prey system with functional response, Math. Biosci. 39 (1978), 124-134.

[10] J. M. McNair, The effects of refuges on predator-prey interactions: a reconsideration, Theor. Popul. Biol. 29 (1986), 38-63.

[11] S. Ruan and D. Xiao, Global analysis in a predator-prey system with nonmonotonic functional response, SIAM J. Appl. Math. 61 (2001), 1445-1472.

[12] G. D. Ruxton, Short term refuge use and stability of predator-prey models, Theor. Popul. Biol. 47(1) (1995), 1-17.

[13] A. Sih, Prey refuges and predator-prey stability, Theor. Popul. Biol. 31(1) (1987), 1-12.

Received April 23, 2019; revised December 14, 2019; accepted December 16, 2019.

\section{Author information}

Unal Ufuktepe, American University of the Middle East, Egaila, Kuwait.

E-mail: Unal. Ufuktepe@aum. edu.kw

Burcin Kulahcioglu, Izmir University of Economics, Izmir, Turkey.

E-mail: burcin.kulahcioglu@ieu.edu.tr 